\title{
Detection of calcium abundance stratification in Ap stars
}

\author{
J. BABEL \\ Service d'Astrophysique, Centre d'Etudes de Saclay, 91191 Gif-sur-Yvette, France
}

\begin{abstract}
We report the discovery of a systematic and large calcium abundance stratification in cold Ap stars. These detections are in very good agreement with diffusion theory and set stringent upper limits on turbulent processes.
\end{abstract}

\section{Context and observations}

The peculiarity of the Ca II K line at $3933 \AA$ (see Fig.1 of Babel 1993b) is a well-known but unexplained feature of Ap stars (since Babcock 1958). On the theoretical side, abundance stratification is a major prediction of radiative diffusion (e.g. Michaud 1970) and has to be tested.

We made a high resolution spectroscopic survey of the $\mathrm{Ca}$ II $\mathrm{K}$ and $\mathrm{H}$ lines at $152 \mathrm{~cm}$ of OHP. It includes $28 \mathrm{Ap}$ stars with $7500 \lesssim T_{\text {eff }} \lesssim 11000$ $\mathrm{K}$ (3 with vsini $\simeq 100 \mathrm{~km} / \mathrm{s}, 2$ rapidly oscillating Ap (roAp)). The $\mathrm{K}$ line profile was parametrized to allow quantitative study of the $\mathrm{K}$ line shape.

Our goal was to discriminate spotted-nonstratified models from stratified models on a statistical ground as any peculiar $\mathrm{K}$ line can be reproduced either by abundance stratification or by abundance spots (Babel 1993a)

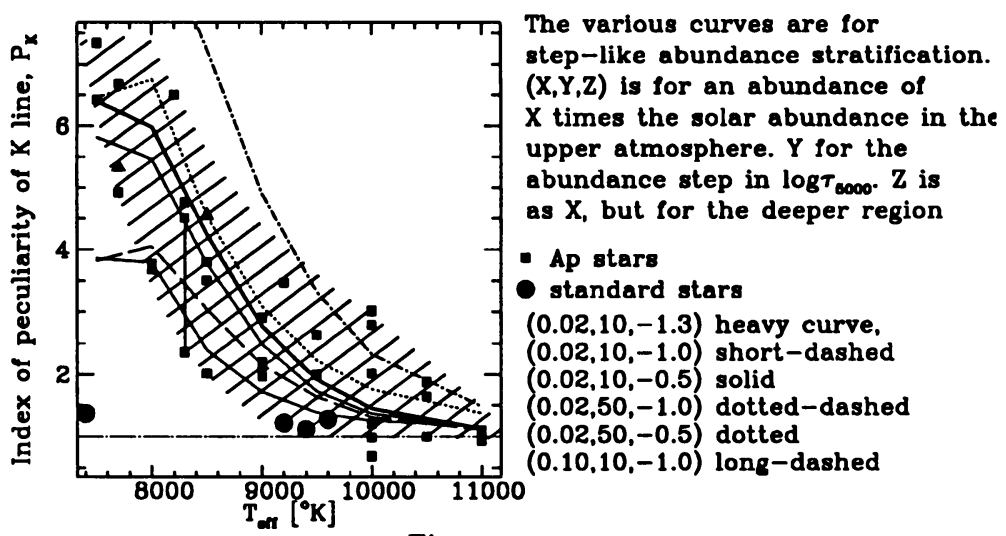

Fig. 1.

\section{Results and Discussion}

In various diagrams relative to the shape of the $\mathrm{K}$ line (see Babel 1993b), Ap stars follow a very different trend than normal stars. In particular, we did not find Ap stars with nonpeculiar profiles, $P_{K} \simeq 1$ for $T_{\text {eff }}<9000 \mathrm{~K}$ 
(Fig. 1). Our results exclude statistically nonstratified-spotted models as an explanation of the peculiar shape of the K line. NLTE effects can also be excluded (Babel 1993b). In contrast, the observations are well explained by a large Ca stratification with decreasing abundance towards the surface.

Stringent additional test comes from the study of the blend $\mathrm{H}_{\epsilon}$-CaII $\mathrm{H}$ and gives another proof of calcium abundance stratification (see Fig. 2.a).
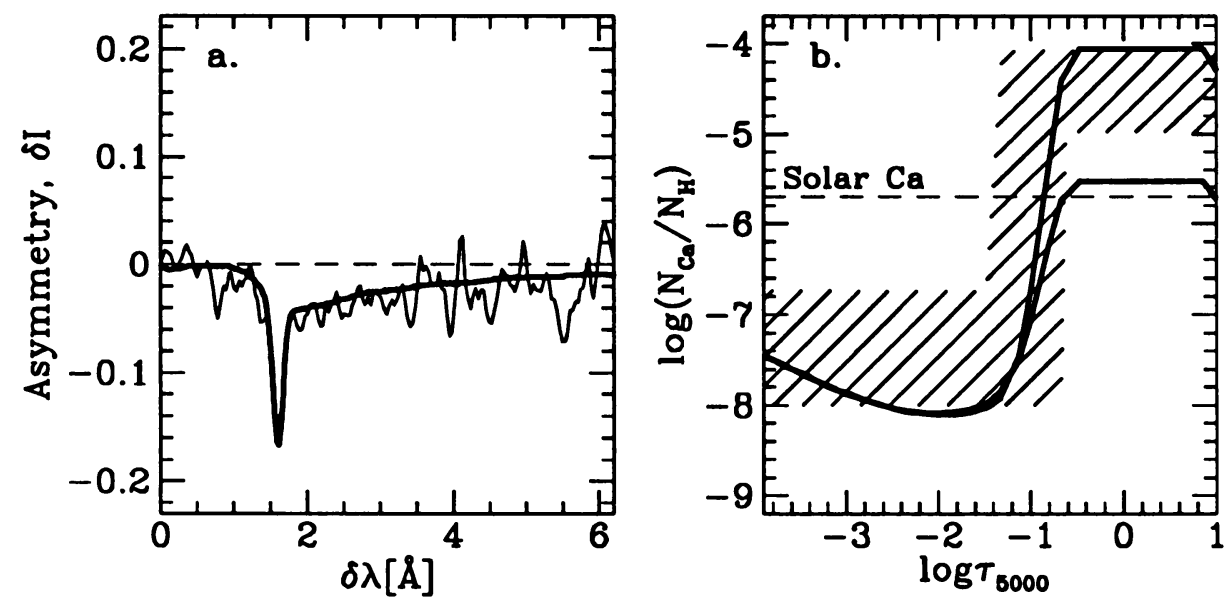

Fig. 2. a.Asymmetry of $H_{\epsilon}$ in $H D 204411$. Heavy line: stratified model $(0.005,10,-1.3)$ (see Babel 1993b). Thin line: observation at DAO by Adelman (private comm.)b. Abundance of $\mathrm{Ca}$ as a function of optical depth. The heavy curves are for the diffusion-mass loss model of 53 Cam with $\dot{M}=310^{-15} \mathrm{M}_{\odot} \mathrm{yr}^{-1}$ (upper curve) and $\dot{M}=0$ (lower curve) (Babel 1992). The hatched zone is the range of stratification of $\mathrm{Ca}$ (step-functions) obtained from the $\mathrm{Ca}$ II $\mathrm{K}$ and $\mathrm{H}$ line for Ap stars with $T_{\text {eff }} \leq 9000 \mathrm{~K}$.

The results on $\mathrm{Ca}$ abundance stratification deduced from the $\mathrm{Ca}$ II $\mathrm{K}$ and $\mathrm{H}$ lines (for an assumed step-function) for Ap stars with $T_{\text {eff }} \lesssim 9000$ $\mathrm{K}$ are summarized by the shaded area in Fig. 3. We obtain that a large Ca stratification, with a variation by 2 dex of the $\mathrm{Ca}$ abundance in the lineformation region, seems very common in Ap with $T_{\text {eff }} \lesssim 9000 \mathrm{~K}$, without effects to first order from rotational velocity or from pulsation for the roAp.

We obtain a very good agreement with equilibrium abundance distributions from the diffusion model (Babel 1992). These results indicate a very large stability of the photospheric regions.

\section{References}

Babel, J.:1992, A\&A 258, 449

Babel, J.:1993a, in Peculiar versus normal phenomena in A-type and related stars, IAU

Coll. 138, Eds. Dworetsky et al., Astron. Soc. Pacific, Conf. Series, 44, p. 458

Babel, J.:1993b, A\&A, in press

Babcock H. W.:1958, ApJS 3, 141

Michaud, G.:1970, ApJ 160, 641 